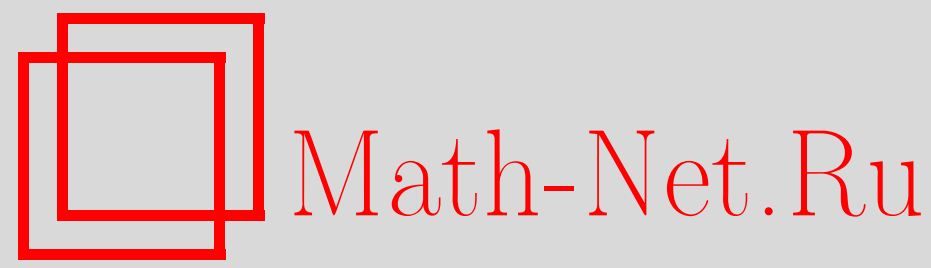

О. Г. Парфенов, Об одном подходе в теории интерполяции в гильбертовых пространствах аналитических функций, Матем. заметки, 1996, том 60, выпуск 5, 780-782

DOI: https://doi.org/10.4213/mzm1893

Использование Общероссийского математического портала Math$\mathrm{Net.Ru}$ подразумевает, что вы прочитали и согласны с пользовательским соглашением

http: //www . mathnet.ru/rus/agreement

Параметры загрузки:

IP : 54.205 .225 .156

26 апреля 2023 г., 16:37:09

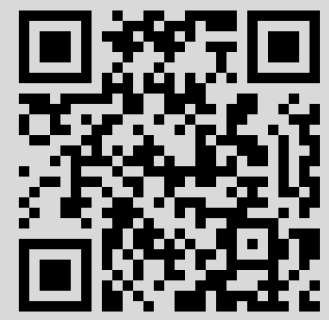




\section{ОБ ОДНОМ ПОДХОДЕ В ТЕОРИИ ИНТЕРПОЛЯЦИИ В ГИЛЬБЕРТОВЫХ ПРОСТРАНСТВАХ АНАЛИТИЧЕСКИХ ФУНКЦИЙ}

\section{О.Г. Парфенов}

1. Пусть $\mathbb{D}$ - единичный круг на комплексной плоскости $\mathbb{C}, H^{2}$ - класс Харди в $\mathbb{D}$. Напомним, что $f \in H^{2}$, если $f$ аналитична в $\mathbb{D}$ и

$$
\|f\|_{H^{2}}^{2}=\sup _{0<r<1} \int_{\mathbb{T}}|f(r z)|^{2} d m(z)<\infty,
$$

$\mathbb{T}$ - единичная окружность, $m$ - мера Лебега на $\mathbb{T}$.

Шапиро и Шилдс в работе [1] доказали очень важньй результат об интерполяции в $H^{2}$. Напомним этот результат. Пусть $E=\left\{z_{n}\right\}_{1}^{\infty}$ - множество в $\mathbb{D}$, причем $\sum_{n=1}^{\infty}\left(1-\left|z_{n}\right|\right)<\infty$. Тем самым определены произведения Бляшке:

$$
B_{n}(z)=\prod_{\substack{k=1 \\ k \neq n}}^{\infty}\left(z-z_{k}\right)\left(1-z \bar{z}_{k}\right)^{-1}\left|z_{k}\right| z_{k}^{-1}, \quad n=1,2, \ldots
$$

Множество Е удовлетворяет условию Карлесона (C), если

$$
\inf _{n}\left|B_{n}\left(z_{n}\right)\right|>0 .
$$

Tеорема 1 [1]. Пусть Е удовлетворяет условию (C). Тогда для любой $f \in H^{2}$

$$
\sum_{n=1}^{\infty}\left|f\left(z_{n}\right)\right|^{2}\left(1-\left|z_{n}\right|^{2}\right)<\infty
$$

Условие (2) называется условием Карлесона-Ньюмена (CN). Теорема Шапиро-Шилдса показьвает, что $(\mathrm{C}) \Longrightarrow(\mathrm{CN})$.

В данной работе приводится новое доказательство этой теоремы, основанное на интерполяции на конечных подмножествах рациональными функциями. План работы такой: в п. 2 мы приводим краткие сведения об интерполяции рациональными функциями в $H^{2}$, в п. 3 дано доказательство теоремы 1, п. 4 содержит некоторые обобщения.

2. Пусть $\left\{z_{k}\right\}_{1}^{N}$ - конечное множество в $\mathbb{D}$. Рассмотрим подпространство в $H^{2}$, натянутое на функции

$$
\varphi_{k}(z)=\left(1-z \bar{z}_{k}\right)^{-1}, \quad k=1,2, \ldots, N .
$$

Обозначим это подпространство $L_{N}$. Обозначим $P_{N}$ ортогоналный проектор в $H^{2}$ на подпространство $L_{N}$. Имеет место

Работа выполнена при поддержке Российского фонда фундаментальных исследований, грант № 95-01-00348. 
Теорема 2 (см. [2]). Oператор $P_{N}$ является интерполячионным, т.е. для любой $f \in H^{2}$

$$
\left(P_{N} f\right)\left(z_{k}\right)=f\left(z_{k}\right), \quad k=1,2 \ldots, N .
$$

Приведем аналог формулы Лагранжа для интерполяции с помощью $L_{N}$. Вместо функций $\varphi_{k}$ удобней взять систему функций

$$
\psi_{k}(z)=\frac{\left(1-\left|z_{k}\right|^{2}\right)^{1 / 2}}{1-z \bar{z}_{k}} \prod_{\substack{i=1 \\ i \neq k}}^{N} \frac{z-z_{i}}{1-z \bar{z}_{i}}=\frac{\left(1-\left|z_{k}\right|^{2}\right)^{1 / 2}}{1-z \bar{z}_{k}} b_{k}(z) .
$$

Имеем

$$
\psi_{k}\left(z_{i}\right)= \begin{cases}0, & k \neq i, \\ c_{k} \neq 0, & k=i .\end{cases}
$$

Отсюда получим представление оператора $P_{N}$ :

$$
\left(P_{N} f\right)(z)=\sum_{k=1}^{N} \frac{f\left(z_{k}\right)}{b_{k}\left(z_{k}\right)}\left(1-\left|z_{k}\right|^{2}\right)^{1 / 2} \frac{b_{k}(z)}{1-z \bar{z}_{k}} .
$$

3. ДОКАЗАТЕЛЬСТВо ТЕОРЕМЫ 1. Достаточно установить, что для множества $\left\{z_{n}\right\}_{1}^{\infty}$, удовлетворяющего условию (C), справедлива равномерная по $N$ оценка

$$
\sum_{n=1}^{N}\left|f\left(z_{n}\right)\right|^{2}\left(1-\left|z_{n}\right|^{2}\right) \leqslant c_{f}<\infty .
$$

Смысл оценки (4) состоит в том, что оператор вложения $J_{N}: H^{2} \rightarrow \ell^{2}\left(E_{N}, \mu_{N}\right)$, $E_{N}=\left\{z_{n}\right\}_{1}^{N}, \mu_{N}\left(z_{n}\right)=\left(1-\left|z_{n}\right|^{2}\right)$, ограничен, и для норм $J_{N}$ имеем

$$
\sup _{n}\left\|J_{N}\right\| \leqslant C<\infty .
$$

Выберем в качестве базиса $H^{2}$ систему функций $\left\{\psi_{k}\right\}_{1}^{N} \cup\left\{g_{n}\right\}_{1}^{\infty},\left\{g_{n}\right\}_{1}^{\infty}$ - базис ядра оператора $J_{N}$. В этом базисе условие (5) принимает вид

$$
\left|\sum_{k, i=1}^{N} \frac{f\left(z_{k}\right) \overline{f\left(z_{i}\right)}}{1-z_{k} \bar{z}_{i}}\left[\left(1-\left|z_{k}\right|^{2}\right)\left(1-\left|z_{i}\right|^{2}\right)\right]^{1 / 2}\right| \leqslant C\|f\|^{2} .
$$

Из теоремы 2 и формулы (3) имеем оценку

$$
\left|\sum_{k, i=1}^{N} \frac{f\left(z_{k}\right) \overline{f\left(z_{i}\right)}}{1-z_{k} \bar{z}_{i}} \frac{\left[\left(1-\left|z_{k}\right|^{2}\right)\left(1-\left|z_{i}\right|^{2}\right)\right]^{1 / 2}}{b_{k}\left(z_{k}\right) \overline{b_{i}\left(z_{i}\right)}}\right| \leqslant\|f\|^{2} .
$$

В силу условия Карлесона и очевидной оценки имеем

$$
\inf _{n}\left|b_{n}\left(z_{n}\right)\right| \geqslant \inf _{n}\left|B_{n}\left(z_{n}\right)\right|>0 .
$$

Из (7) и (8) следует (6). 
4. В данном пункте приведем обобщение теоремы 2 на любые гильбертовы пространства с воспроизводящим ядром. Напомним, что функциональное гильбертово пространство $X^{2}(G)$ имеет воспроизводящее ядро $R(z, w)$, если для $z \in G$ и любой $f \in X^{2}(G) f(z)=(f(\cdot), R(\cdot, z))_{X^{2}(G)}$. Например, для $H^{2}$ воспроизводящее ядро есть функция $(1-z \bar{w})^{-1}$.

Пусть $\left\{z_{k}\right\}_{1}^{N}$ - произвольное множество в $G$. Рассмотрим подпространство в $X^{2}(G)$, натянутое на систему функций $\left\{R\left(\cdot, z_{k}\right)\right\}_{1}^{N}$. Обозначим это подпространство $L_{N}$. Обозначим $P_{N}$ ортопроектор в $X^{2}(G)$ на $L_{N}$.

ТеОрема 3. Oператор $P_{N}$ является интерполяционным, т.е. для любой $f \in X^{2}(G)$

$$
\left(P_{N} f\right)\left(z_{k}\right)=f\left(z_{k}\right), \quad k=1,2, \ldots, N .
$$

ДокАЗАТЕЛЬСтво. Пусть $I_{N} f$ - интерполяционный многочлен $f$ по системе функций $\left\{R\left(\cdot, z_{k}\right)\right\}_{1}^{N}$. В силу линейной независимости этой системы многочлен $I_{N} f$ существует и притом единственный. Имеем

$$
\left(I_{N} f-f, R\left(\cdot, z_{k}\right)\right)_{X^{2}(G)}=\left(I_{N} f\right)\left(z_{k}\right)-f\left(z_{k}\right)=0,
$$

$k=1, \ldots, N$, что и доказывает теорему 3 .

СледСтвИЕ. Пусть $\left\{z_{k}\right\}_{1}^{\infty}$ - мнохество единственности для $X^{2}(G)$, m.е. из того, ито $f \in X^{2}(G)$ и $f\left(z_{k}\right)=0, k=1,2, \ldots$, следует, ито $f=0$. Тогда последовательность интерполячионних многочленов $\left\{I_{N} f\right\}_{N=1}^{\infty}$ сходится $\kappa f$ в $X^{2}(G)$ и, следовательно, равномерно внутри $G$.

В частности, для $H^{2}$ получим такой результат.

Tеорема 4. Пусть $\left\{z_{n}\right\}_{1}^{\infty}$ - мнохсество в $\mathbb{D} u\left\{I_{N} f\right\}_{1}^{\infty}$ - последовательность интерполяционных многочленов некоторой функции $f \in H^{2}$, построенная по формуле (3).

а) $\operatorname{Ec\Omega u~} \sum_{n=1}^{\infty}\left(1-\left|z_{n}\right|\right)=\infty$, mo $I_{N} f \rightarrow f$ nрu $N \rightarrow \infty$ в пространстве $H^{2}$.

б) $\operatorname{Eсли~} \sum_{n=1}^{\infty}\left(1-\left|z_{n}\right|\right)<\infty$ и B(z) - произведение Бляшке, построенное по узлам $\left\{z_{n}\right\}_{1}^{\infty}, L=B H^{2}$ и $P$ - ортопроектор на подпространство $H^{2} \ominus B H^{2}$, mo $I_{N} f \rightarrow \operatorname{Pf}$ при $N \rightarrow \infty$ в пространстве $H^{2}$.

\section{СПИСОК ЦИТИРОВАННОЙ ЛИТЕРАТУРЫ}

1. Shapiro Y. S., Shields A. L. // Amer. J. Math. 1961. V. 83. № 2. P. 513-532. 2. Гайер Д. Лекции по теории аппроксимации в комплексной области. М.: Мир, 1986. 